\title{
Radially patterned polycaprolactone nanofibers as an active wound dressing agent
}

\author{
Dongwoo Shin ${ }^{1}$, Min Sup Kim ${ }^{2}$, Chae Eun Yang ${ }^{3}$, Won Jai Lee ${ }^{1}$, Tai Suk Roh ${ }^{1}$, Wooyeol Baek ${ }^{1}$ \\ ${ }^{I}$ Department of Plastic and Reconstructive Surgery, Institute for Human Tissue Restoration, Severance Hospital, Yonsei University College of \\ Medicine, Seoul; ${ }^{2}$ PLCOSKIN Inc., Seoul; ${ }^{3}$ Department of Plastic and Reconstructive Surgery, Wonju Severance Christian Hospital, Yonsei \\ University Wonju College of Medicine, Wonju, Korea
}

Background The objectives of this study were to design polycaprolactone nanofibers with a radial pattern using a modified electrospinning method and to evaluate the effect of radial nanofiber deposition on mechanical and biological properties compared to non-patterned samples.

Methods Radially patterned polycaprolactone nanofibers were prepared with a modified electrospinning method and compared with randomly deposited nanofibers. The surface morphology of samples was observed under scanning electron microscopy (SEM). The tensile properties of nanofibrous mats were measured using a tabletop uniaxial testing machine. Fluorescence-stained human bone marrow stem cells were placed along the perimeter of the radially patterned and randomly deposited. Their migration toward the center was observed on days 1, 4, and 7, and quantitatively measured using ImageJ software.

Results Overall, there were no statistically significant differences in mechanical properties between the two types of polycaprolactone nanofibrous mats. SEM images of the obtained samples suggested that the directionality of the nanofibers was toward the central area, regardless of where the nanofibers were located throughout the entire sample. Florescence images showed stronger fluorescence inside the circle in radially aligned nanofibers, with significant differences on days 4 and 7, indicating that migration was quicker along radially aligned nanofibers than along randomly deposited nanofibers.

Conclusions In this study, we successfully used modified electrospinning to fabricate radially aligned nanofibers with similar mechanical properties to those of conventional randomly aligned nanofibers. In addition, we observed faster migration along radially aligned nanofibers than along randomly deposited nanofibers. Collectively, the radially aligned nanofibers may have the potential for tissue regeneration in combination with stem cells.

Keywords Polycaprolactone / Polymers / Nanofibers / Wound healing
Correspondence: Wooyeol Baek Department of Plastic and Reconstructive Surgery, Institute for Human Tissue Restoration, Severance Hospital, Yonsei University College of Medicine, 50 Yonsei-ro, Seodaemungu, Seoul 03722, Korea

Tel: +82-2-2228-2220

Fax: +82-2-393-6947

E-mail: PARANDE@yuhs.ac
This research was supported by Basic Science Research Program through the National Research Foundation of Korea (NRF) funded by the Ministry of Education (NRF2018R1D1A1B07051132). This research was also supported by the Bio \& Medical Technology Development Program of the NRF funded by the Korean government (MSIT) (NRF-2019M3A9E8022138), and by a faculty research grant of Yonsei University College of Medicine (6-20180094).

\section{INTRODUCTION}

Nanofibers are regarded as valuable materials for research in the field of tissue regeneration, as they can physically support the cells that constitute the human body and control the storage and release of various types of functional proteins that regulate

Copyright (C) 2019 The Korean Society of Plastic and Reconstructive Surgeons

This is an Open Access article distributed under the terms of the Creative Commons Attribution Non-Commercial License (http://creativecommons.org/

licenses/by-nc/4.0/) which permits unrestricted non-commercial use, distribution, and reproduction in any medium, provided the original work is properly cited. I www.e-aps.org 
cell metabolism; furthermore, they have a similar structure to that of the extracellular matrix, allowing them to function as a matrix for cell culture [1]. However, due to the fact that they have a thin nonwoven fabric form, with a thickness of less than $100 \mu \mathrm{m}$, they have the limitation of being vulnerable to pressure and failure to incorporate into the surrounding tissue inside the body. However, in the field of plastic surgery, nanofibers based on biodegradable polymers with high elasticity can be an appropriate material for promoting skin regeneration. They are often fabricated using the electrospinning technique, which has the advantages of cost-effectiveness, procedural simplicity, and versatility. Electrospinning allows nanofibers to have a uniform and continuous structure with a controllable pore structure, resulting in high porosity and a large surface-to-volume ratio [2]. Their structure enables exchange of fluid and gas, absorbance of excess exudates and wound secretions, and cell adherence and growth [3]. Therefore, clinical scientists are actively studying nanofibers combined with functional proteins, drugs, metals, ceramics, and surface modifications for application as functional wound healing agents.

Common biodegradable polymers used for electrospun nanofibers include polylactic acid (PLA), polyglycolic acid (PGA), and polycaprolactone (PCL), which have versatile mechanical performance and good spinnability [4]. During the process of degradation, PLA and PGA are known to cause inflammation by making the surrounding tissue weakly acidic, whereas PCL is completely degraded through the citric acid cycle. In addition, since PCL is a polymer with high elasticity, it could potentially be a suitable wound healing agent for covering a large area of skin. PCL is degraded in vivo by microorganisms, at a slower rate (up to 3-4 years) than other biodegradable materials, such as PLA and PGA [3]. Its slower biodegradation makes PCL more suitable for long-term application as a biomaterial than other polymers. PCL also has the advantages of ease of manufacture and manipulation, making it possible to render it into various forms such as nanospheres, nanofibers, and foams at a low cost [5-7]. For these reasons, we selected PCL as a biodegradable polymer for the nanofibers.

The physical and biological properties related to the alignment of PCL nanofibers remain poorly understood. The role of the orientation of PCL nanofibers in stem cell migration is also still unclear. Although several studies have investigated the alignment of nanofibers, those studies were only confined to linearly aligned nanofibers. We hypothesized that radially aligned nanofibers would induce the migration of fibroblasts toward the center, thereby resulting in faster and more stable wound healing. The objectives of this study were to design cell culture substrates with radially patterned PCL nanofibers using a modified electrospinning method and to evaluate the effects of those patterns on the mechanical and biological properties of the substrates compared to non-patterned samples of PCL polymers.

\section{METHODS}

To prepare the radially patterned samples, a rotating collector was covered by a metal pin and stainless ring (diameter, $15 \mathrm{~mm}$ ) attached to an insulating plate. Then, $6 \%$ by weight PCL solution dissolved in hexafluoroisopropanol was ejected using a syringe pump (ES-Bio, Nanao NC, Seoul, Korea) toward the collector at a flow rate of $1 \mathrm{~mL} / \mathrm{hr}$ (Fig. 1). The samples were then

Fig. 1. Schematic illustration of the modified electrospinning apparatus

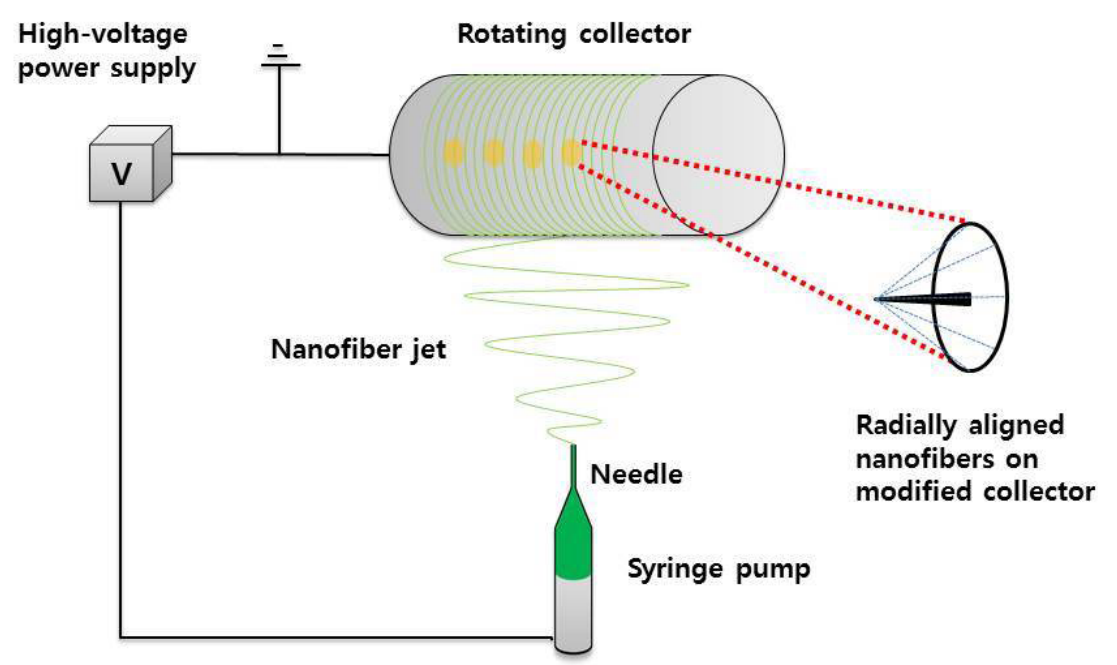


dried overnight and prepared by wetting with $70 \%$ ethanol. The surface morphology of samples was observed with a scanning electron microscopy (SEM; Gemini 2, Zeiss, Oberkochen, Germany). To measure the distribution of the diameter and pore size of nanofibers, 10 fields from each image were selected and the fiber diameters were manually measured using a ruler generated within the image analyzer (Image Pro-Plus; Media Cybernetics Inc., Rockville, MD, USA). The tensile properties of the nanofibrous mats (thickness, $70-80 \mu \mathrm{m}$ measured by a $7304 \mathrm{~S}$ thickness gauge; Mitutoyo, Kawasaki, Japan) were measured using a tabletop uniaxial testing machine (Instron5567; Canton, MA, USA) with a cross-head speed of $1 \mathrm{~mm} / \mathrm{min}$ under ambient conditions. Cellular migration on the mats was determined by tracing cell-free areas under in vitro conditions. To fabricate a cell-free area on the mats, polydimethylsiloxane cylinders with a 6-mm diameter were placed on the mat, and then human bone marrow cell stem cells (passage no. 6; Lonza, Basel, Switzerland) were seeded on the perimeter of both randomly aligned $(n=4)$ and radially aligned $(n=4)$ nanofibrous mats at a density of 10,000 cells/sample. The human bone marrow stem cells were cultured in DMEM (Gibco BRL; Carlsbad, CA, USA) supplemented with $10 \%$ fetal bovine serum and $1 \%$ penicillinstreptomycin under standard culture conditions $\left(37^{\circ} \mathrm{C}, 5 \%\right.$ $\mathrm{CO}_{2}$ ) and stained with Alexa Fluor 488, a dye specific for actin filaments. Their migration toward the center was observed under the microscope and compared at days 1,4 , and 7 . The cellfree area on the image was measured using ImageJ software (NIH, Bethesda, MD, USA).

Quantitative data were obtained in quadruplicate and are reported as median, maximum and minimum values, as indicated. Statistical analyses were performed using the Mann-Whitney U test for non-parametric testing between two groups. A P-value $<0.1$ was considered to indicate statistical significance.

\section{RESULTS}

The tensile properties of samples were analyzed using a uniaxial testing machine. The Young modulus, elongation percentage, and tensile strength were measured (Table 1 ). The radially aligned samples showed slightly lower values than the random samples. Overall, there was no statistically significant difference in the mechanical properties between the two different types of PCL nanofibrous mats.

We observed morphological differences between the radially aligned and randomly deposited nanofibrous mats using SEM analysis. SEM images of the radially aligned nanofiber samples were observed, and suggested that directionality of the nanofibers was toward the central area, regardless of where the nanofi-

Table 1. Comparison of tensile properties between randomly deposited and radially aligned nanofibers

\begin{tabular}{|lccc|}
\hline & Random $(\mathbf{n}=4)$ & Radial $(\mathbf{n}=4)$ & P-value \\
\hline Young modulus (MPa) & $21.43(16.71-26.75)$ & $20.37(13.18-25.41)$ & 0.564 \\
Elongation (\%) & $146.75(129.42-172.61)$ & $159.30(133.46-178.67)$ & 0.564 \\
Tensile strength (MPa) & $15.03(11.75-17.36)$ & $10.65(9.15-13.51)$ & 0.486 \\
\hline Values are presented as median (range). & & & \\
\hline
\end{tabular}

\section{Fig. 2. SEM images of the PCL nanofiber}

(A) Randomly deposited polycaprolactone (PCL) nanofibers. (B) Radially aligned PCL nanofibers (red arrow: fiber direction). SEM, scanning electron microscopy.
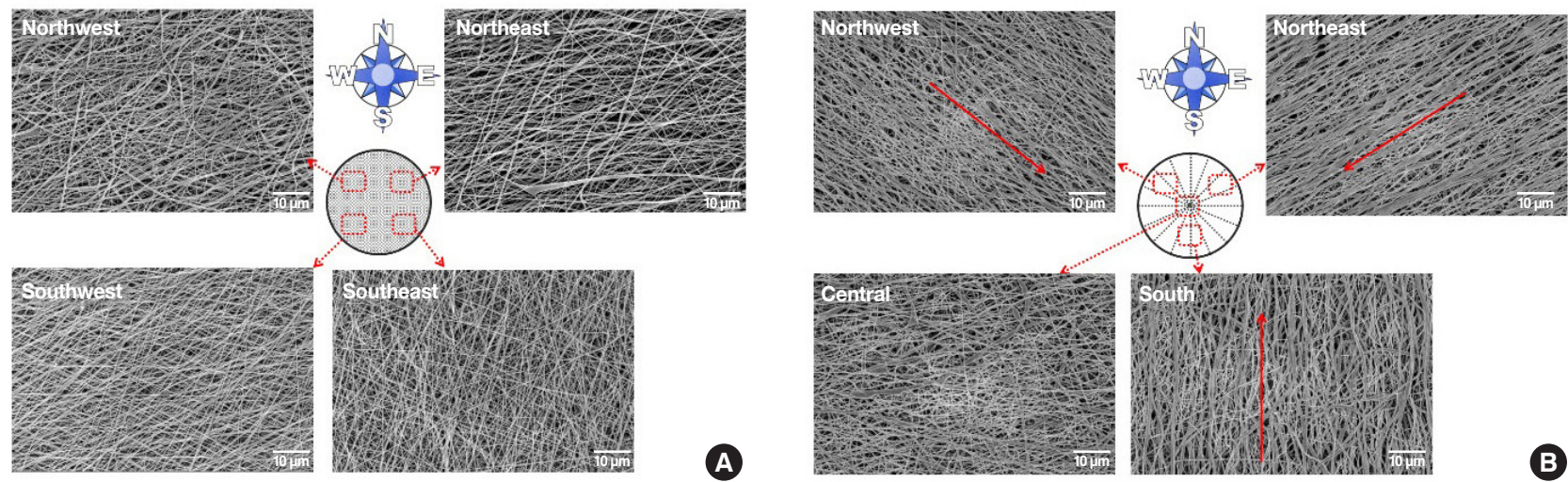
bers were located throughout the entire sample. In contrast, no directionality was observed in the randomly deposited PCL nanofibers (Fig. 2). The average diameter of the samples was calculated to be $374.00 \pm 27.13 \mathrm{~nm}$ and $391.12 \pm 17.15 \mathrm{~nm}$ for the radially aligned and randomly deposited nanofibrous mats, respectively. Furthermore, the pore size between fibers was measured as $11.45 \pm 3.84 \mathrm{~nm}$ and $12.71 \pm 4.76 \mathrm{~nm}$ for the radially aligned and randomly deposited nanofibrous mats, respectively. In the morphological analysis, the only difference between these samples was the directionality of the nanofibers. Photomicrographs of the fluorescence-stained bone marrow stem cells were obtained on days 1, 4, and 7 (Fig. 3). On the images from both groups, more bone marrow stem cells were observed inside the nanofibrous mats. Cells gradually migrated into the center of the nanofibrous mat over time. They showed stronger fluorescence inside the circle in the radially aligned nanofibrous mats than in the randomly distributed nanofibrous mats. Measurements of the proportion of cell-free areas showed statistically significant differences on both day 4 and 7 between the two types of mats. On day 4, the median cell-free area was $86.4 \%$, ranging from $78.7 \%$ to $93.4 \%$ in the randomly deposited mats, and a median of $60.6 \%$, ranging from $51.7 \%$ to $75.5 \%$, in the radially aligned nanofibrous mats. On day 7 , the median cell-free area was $84.1 \%$, ranging from $51.7 \%$ to $89.9 \%$, in the randomly deposited nanofibrous mats, and a median of $48.9 \%$, ranging from $43.6 \%$ to $57.2 \%$, in the radially aligned nanofibrous mats (Fig. 4). This result indicates that cellular migration was more rapid along the radially aligned nanofibers than along the randomly deposited nanofibers.

\section{Fig. 3. Cell migration speed difference}

Photomicrographs of fluorescence-stained bone marrow stem cells in randomly deposited nanofibrous mats and radially aligned nanofibrous mats on days 1,4 , and 7 .

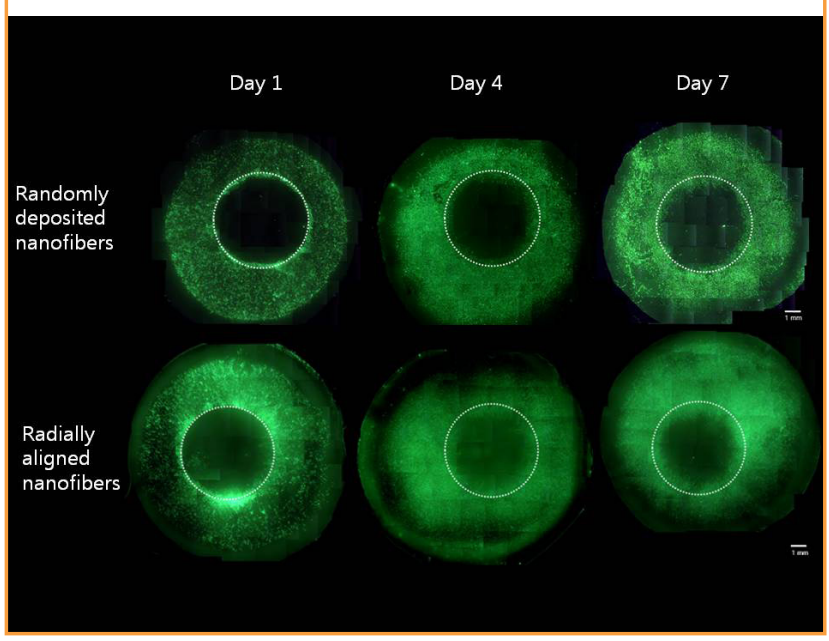

\section{DISCUSSION}

Several studies have investigated alterations in the alignment of PCL and analyzed the properties of PCL according to its structure. Among the numerous relevant technologies that have been developed, electrospinning is inexpensive way of manufacturing submicron- and micron-diameter fibers from PCL polymer solutions. Zhang et al. [8] compared electrospun PCL fibers to solid-form PCL in terms of the recruitment of mesenchymal stem cells (MSCs) and found that fibrous structures enhanced the recruitment of MSCs by promoting macrophage recruitment, facilitating the phenotypic transition, and enhancing chemokine protein secretion. Recently, Bhullar et al. [9] generated auxetic PCL nanofibers using electrospinning and a laser-cut technique. They revealed that the auxetic PCL fibers had similar chemical functionality and thermal properties, but a 10 -fold higher elongation capacity than conventional PCL nanofibers.

In this study, we successfully generated radially aligned nanofibers using a modified electrospinning method. We confirmed the morphological difference between the sample surfaces using SEM. This study demonstrated that PCL nanofibers with different types of alignment can be fabricated by electrospinning. Additionally, these radially aligned PCL nanofibers had similar physical properties to those of non-aligned PCL nanofibers, indicating that radially aligned PCL nanofibers can be readily used in place of conventional PCL, with proper structural and mechanical function.

We observed faster migration of stem cells along radially aligned nanofibers than along randomly deposited nanofibers.

\section{Fig. 4. The proportion of cell-free area}

Comparison of the proportion of the cell-free area between randomly deposited nanofibers and radially aligned nanofibers on days 1,4 , and 7. Values are presented as median (range). ${ }^{a} \mathrm{P}=0.029$; b) $P=0.057$.

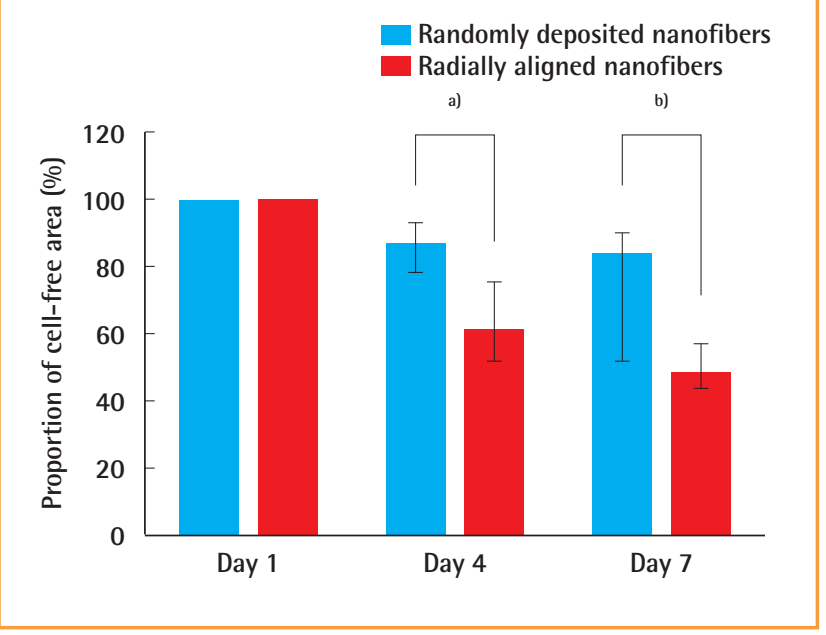


A previous study proved that the topographical structure of PCL influenced the migration of stem cells, with fibrous structures showing faster migration than solid forms [8]. Our study further revealed that radial alignment of PCL facilitated enhanced migration. Therefore, radially aligned PCL nanofibers may serve as a potential material for various biomedical applications — in particular, tissue engineering — as scaffolds or active tissue dressing material for soft tissue defects. We can assume that endogenous stem cells in the applied area would migrate faster, thereby enhancing tissue ingrowth.

Exogenous bone marrow stem cells themselves can be used as a way to regenerate and repair defective tissue. MSCs have been used to accelerate tissue healing due to their high self-renewal capacity and excellent differentiation potential $[10,11]$. A challenge for cell-based therapies is to achieve effective and optimal targeted delivery and engraftment of cells at the site of injury, especially MSCs homing to sites of injury [5]. Our study suggested that it may be possible to guide cells to the injured area by modifying the structure of PCL fibers. With the development of tissue engineering, radially aligned PCL nanofibers eventually might be able to serve as wound-dressing agents that provide effective scaffolds for use with stem cells.

PCL nanofibers can be applied not just with stem cells, but also with other substances such as cytokines, growth factors, or therapeutic drugs. Some cytokines are known to promote the recruitment of stem cells. For example, SDF-1, which is a wellknown chemokine, has been shown to recruit MSCs through its interaction with CXC chemokine receptors on the surface of MSCs [6]. Sawyer et al. [7] reported that PCL, tricalcium phosphate (TCP), and collagen with recombinant human bone morphogenetic proteins stimulated healing compared to PCL, TCP, and collagen alone in rat calvarial defects. When used as an external dressing material, nanofibers could be used as a release vehicle for the chemical antiseptic chlorohexidine [12]. Multifunctional electrospun nanofibrous mats of mussel adhesive protein and PCL accelerated regeneration in a rat skin wound-healing model [13].

However, this experiment was limited in that it was done only using in vitro culture. Future studies should be conducted to observe how actual host tissue interacts with radially aligned PCL in the setting of in vivo implantation. The biosorption, biocompatibility, and healing process of these fibers inside host tissue remain to be studied in both short-term and long-term periods.

In this study, we successfully fabricated radially aligned nanofibers with similar mechanical properties to those of conventional randomly aligned nanofibers using modified electrospinning. In addition, we observed faster migration along radially aligned nanofibers than along randomly deposited nanofibers. Overall, radially aligned nanofibers may have potential for tissue regeneration in combination with stem cells.

\section{NOTES}

\section{Conflict of interest}

No potential conflict of interest relevant to this article was reported.

\section{Author contribution}

Conceptualization: Baek W. Data curation: Kim MS. Formal analysis: Kim MS, Shin D. Funding acquisition: Roh TS. Methodology: Kim MS. Project administration: Lee WJ. Visualization: Kim MS. Writing - original draft: Kim MS. Writing - review \& editing: Baek W, Yang CE. Approval of final manuscript all authors.

\section{ORCID}

Dongwoo Shin https://orcid.org/0000-0002-6081-9646

Min Sup Kim https://orcid.org/0000-0002-4212-2490

Chae Eun Yang https://orcid.org/0000-0001-8128-791X

Won Jai Lee https://orcid.org/0000-0003-3056-0503

Tai Suk Roh https://orcid.org/0000-0001-8681-159X

Wooyeol Baek https://orcid.org/0000-0002-6638-4110

\section{REFERENCES}

1. Bhardwaj N, Kundu SC. Electrospinning: a fascinating fiber fabrication technique. Biotechnol Adv 2010;28:325-47.

2. Liu M, Duan XP, Li YM, et al. Electrospun nanofibers for wound healing. Mater Sci Eng C Mater Biol Appl 2017;76: 1413-23.

3. Yang Y, Xia T, Zhi W, et al. Promotion of skin regeneration in diabetic rats by electrospun core-sheath fibers loaded with basic fibroblast growth factor. Biomaterials 2011;32: 4243-54.

4. Liang D, Hsiao BS, Chu B. Functional electrospun nanofibrous scaffolds for biomedical applications. Adv Drug Deliv Rev 2007;59:1392-412.

5. Karp JM, Leng Teo GS. Mesenchymal stem cell homing: the devil is in the details. Cell Stem Cell 2009;4:206-16.

6. Marquez-Curtis LA, Janowska-Wieczorek A. Enhancing the migration ability of mesenchymal stromal cells by targeting the SDF-1/CXCR4 axis. Biomed Res Int 2013;2013: 561098.

7. Sawyer AA, Song SJ, Susanto E, et al. The stimulation of healing within a rat calvarial defect by $\mathrm{mPCL}-\mathrm{TCP} /$ collagen scaffolds loaded with rhBMP-2. Biomaterials 2009;30: 
2479-88.

8. Zhang Q, Hwang JW, Oh JH, et al. Effects of the fibrous topography-mediated macrophage phenotype transition on the recruitment of mesenchymal stem cells: an in vivo study. Biomaterials 2017;149:77-87.

9. Bhullar SK, Rana D, Lekesiz H, et al. Design and fabrication of auxetic PCL nanofiber membranes for biomedical applications. Mater Sci Eng C Mater Biol Appl 2017;81:334-40.

10. Nakajima H, Uchida K, Guerrero AR, et al. Transplantation of mesenchymal stem cells promotes an alternative pathway of macrophage activation and functional recovery after spinal cord injury. J Neurotrauma 2012;29:1614-25.
11. Lee RH, Pulin AA, Seo MJ, et al. Intravenous hMSCs improve myocardial infarction in mice because cells embolized in lung are activated to secrete the anti-inflammatory protein TSG-6. Cell Stem Cell 2009;5:54-63.

12. Medlicott NJ, Jones DS, Tucker IG, et al. Preliminary release studies of chlorhexidine (base and diacetate) from poly ( $\epsilon$-caprolactone) films prepared by solvent evaporation. Int J Pharm 1992;84:85-9.

13. Kim BJ, Cheong H, Choi ES, et al. Accelerated skin wound healing using electrospun nanofibrous mats blended with mussel adhesive protein and polycaprolactone. J Biomed Mater Res A 2017;105:218-25. 